\title{
Emotion Classification in Arousal Valence Model using MAHNOB-HCI Database
}

\author{
Mimoun Ben Henia Wiem \\ Université de Tunis El Manar, \\ Ecole Nationale d'Ingénieurs de Tunis, \\ LR-11-ES17, Signal, Images et Technologies de \\ l'Information (LR-SITI-ENIT) \\ BP. 37 Belvédère, 1002, Tunis, Tunisie
}

\author{
Zied Lachiri \\ Université de Tunis El Manar, \\ Ecole Nationale d'Ingénieurs de Tunis, \\ LR-11-ES17, Signal, Images et Technologies de \\ l'Information (LR-SITI-ENIT) \\ BP. 37 Belvédère, 1002, Tunis, Tunisie
}

\begin{abstract}
Emotion recognition from physiological signals attracted the attention of researchers from different disciplines, such as affective computing, cognitive science and psychology. This paper aims to classify emotional statements using peripheral physiological signals based on arousal-valence evaluation. These signals are the Electrocardiogram, Respiration Volume, Skin Temperature and Galvanic Skin Response. We explored the signals collected in the MAHNOB-HCI multimodal tagging database. We defined the emotion into three different ways: two and three classes using 1-9 discrete self-rating scales and another model using 9 emotional keywords to establish the three defined areas in arousal-valence dimensions. To perform the accuracies, we began by removing the artefacts and noise from the signals, and then we extracted 169 features. We finished by classifying the emotional states using the support vector machine. The obtained results showed that the electrocardiogram and respiration volume were the most relevant signals for human's feeling recognition task. Moreover, the obtained accuracies were promising comparing to recent related works for each of the three establishments of emotion modeling.
\end{abstract}

Keywords-Emotion Classification; MAHNOB-HCI; Peripheral Physiological Signals; Arousal-Valence Space; Support Vector Machine

\section{INTRODUCTION}

For affective and correct interaction between human and machine (HCI), recognizing human's emotion is a one of the key stage in affective computing field and especially in emotional intelligence for HCI issue. Thus, several researches could be targeted that will benefit from feeling assessment. We cite those done in medicine field and particularly for children with autism who are disable to clearly express their feelings [1]. Emotion recognition system can identify the critical states during driving by detecting the stress level assessments [2][3][4]. Moreover, there are applications that affect daily lives without stress[5] with more pleasing life[6].

The emotion can be noticeable from different modalities. The facial expression is the most popular way to recognize the affective states [7][8][9]. Also, the human speech [10][11] and motions or gestures are very used in emotion assessing problem. However, these channels cannot usually identify the real emotional states because it is easy to secret a facial expression or fake a tone of voice[12]. Moreover, they are not effective for people who cannot reveal their feeling verbally like autistic people [13]. Also, they aren't available unless the user is usually facing to the camera or microphone in an adequate environment with no dark or noise for data collection. To proceed these problems, Picard et al. [14] demonstrated that physiological signals are more pertinent than other modalities. In fact, they originate from the peripheral nervous system and central nervous system. Consequently, they cannot be falsified or hidden. Moreover, they are spontaneous and strongly correlated with human's emotion. The physiological signals are (1) Electroencephalograms (EEG), (2) Electrocardiogram (ECG), (3) Heart Rate Variability (HRV), (4) Galvanic Skin Response (GSR), (5) Muscle Activity or Electromyogram (EMG), (6) Skin Temperature (SKT), (7) Blood Volume Pulse (BVP) and (8) Respiratory Volume (RESP). Since, many studies became very enhanced in emotion recognition problem [15][16][17] and recent works combine physiological signals with two or more modalities to improve the results [18][19].

It is difficult to compare these investigated approaches because they are divergent in different ways. Indeed, the related works are dissimilar in the modality to recognize the affective states that can be natural or induced. Thus, emotion can be evoked by watching affective movies [20], video clips [21], since playing a video game [22], driving a car or listening to music [23][24]. Moreover, the emotion can be defined into different models: the first is Eckman's model that is based on universal emotional expressions to present out six discrete basic emotions: Happiness, Sadness, Surprise, Fear, Anger and Disgust [25]. The second is the Plutchik's model that presents out eight fundamental emotions: Joy, Trust, Fear, Surprise, Sadness, Disgust, Anger and Anticipation [26]. The third is based on Russel et al. model [27] who have focused on twodimensional evaluation, like the valence-arousal model [19]. Some other works merge the previous models to define the emotion in the continuous space using affective keywords [28][20].

Among recent and related researches, we cite the work based on MAHNOB dataset [20]. They classified the affective states into three defined classes and they achieved $46.2 \%$, $45.5 \%$ for arousal and valence, respectively. Another similar work done by Koelstra et al. who created freely multimodal dataset DEAP, is detailed in [21]. They classified the emotional statements into two classes for valence, arousal and liking. In the previous contribution, they obtained $57 \%, 62.7 \%, 59.1 \%$ for arousal, valence and liking, respectively. We notice that we cannot directly compare these studies, because they used 
different classes in arousal valence model. Moreover, the manner to define the emotion is dissimilar: In fact, in [20], they classified the affective statements using nine discrete emotional keywords tagging to define three classes in arousal valence model. However, in [21], they used discrete self-rating scales from 1 to 9 for arousal, valence and liking.

This paper aims to identify the human affective states into arousal-valence area using three ways of modeling and defining the emotion in this continuous space. The proposed approach is based on peripheral physiological signals (ECG, Resp, Temp and GSR) to use wearable and non-obtrusive sensors for future work. We began by defining the emotional states into two classes which are "positive" and "negative" for valence and "High" and "Low" in arousal. Then, we established three classes using the self-reported discrete scaling values (from 1to 9 scales in arousal and valence axis). The three classes are named calm, medium aroused, and excited, unpleasant, neutral valence and pleasant). Finally, we defined these three classes using nine emotional keywords (Happiness, amusement, neutral, anger, fear, surprise, anxiety, disgust and sadness). In the last emotion's definition, we combined the emotion's model done by Russel and Ekman [28][29][30]. Another purpose of this paper is to select the most relevant peripheral physiological signal for emotion sensing problem. Firstly, we began by classifying one signal and then, we fused their level features. We explored the recent multimodal MAHNOB-HCI database. A judiciously process was applied in preprocessing, features extraction and classification stages. For the last step we used the support vector machine (SVM).

The foregoing of this paper is organized as follows. Section II describes the proposed approach. The next section gives the details of the preprocessing data and feature extraction stages. In section IV, we present SVM classifier and how we modeled the emotion states. The section $\mathrm{V}$ summarizes the obtained results. Finally, we conclude this contribution and present future work in section VI.

\section{EXPERIMENTAL SETUP}

\section{A. Proposed approach}

The emotion recognition system had several steps that ought to be carefully done to have promising classification rate. As a first step, we pre-processed the data to smooth the signals. Then, a bunch of selected features were extracted. After normalizing all features, an early level feature fusion (LFF) was applied to compare the proposed approach to related works. Finally, we classified the data corresponding to their labels using the support vector machine. All these steps will be detailed in the foregoing sections. Fig .1 presents the block diagram of this work.

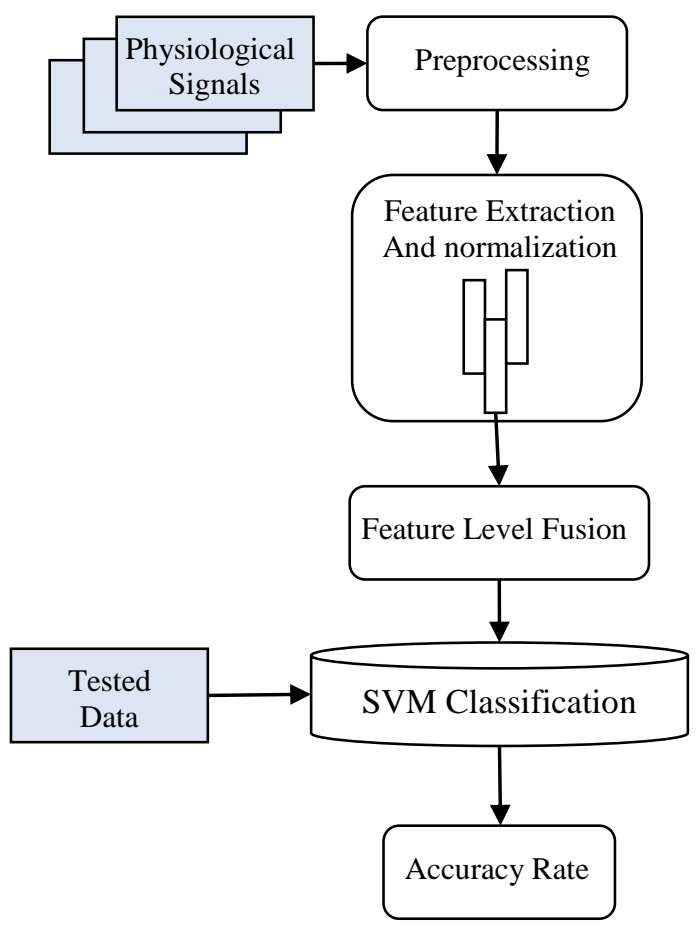

Fig. 1. Block diagram of the proposed approach

\section{B. MAHNOB-HCI Multimodal Database}

Investigations in emotion recognition field motivated the establishment of many databases to involve this issue. Some datasets contained speech and audio-visual signals as modalities to assess the human affective states [31][32]. Healey and Picard [33] collected one of the first affective physiological datasets at MIT. Their collected signals were the electrocardiogram (ECG), galvanic skin response (GSR), electromyogram (EMG) and the respiration pattern. This database of stress recognition is publicly available from Physionet ${ }^{1}$. Another novel dataset is the Database for Emotion Analysis using Physiological signals (DEAP) [21]. It contains the spontaneous bodily responses of 32 participants after inducing their emotional states by watching selected music videos clips. This dataset is freely available on the internet ${ }^{2}$ for academic research. More recently, Soleymani et al.[20] created the MAHNOB-HCI multimodal database. They recorded the peripheral physiological signals from 24 participants after eliciting their emotion by 20 affective movies. These signals are the Electrocardiogram (ECG), Galvanic Skin Response (GSR), Skin Temperature (Temp) and Respiration Volume (RESP). They also recorded the EEG signal, eye gaze and the face videos. 
In the proposed approach, we chose the latest database for several reasons. In fact, it had five modalities which were judiciously synchronized. Also, a comparative study between DEAP and MAHNOB datasets done by Godin et al. [34], demonstrated that the best accuracies were obtained after using the recorded signals in MAHNOB database. Table .I summarizes the content of the MAHNOB-HCI database.

TABLE I. DATABASE SUMmary[20]

\begin{tabular}{|l|l|}
\hline $\begin{array}{l}\text { Number of } \\
\text { participants }\end{array}$ & 24 \\
\hline Recorded signals & $\begin{array}{l}\text { Peripheral physiological signals 256Hz (ECG, GSR, } \\
\text { Temp, Resp), Face and body video using 6 cameras } \\
(60 \mathrm{f} / \mathrm{s}), 32 \text { channels for EEG signal }(256 \mathrm{~Hz}), \text { Eye } \\
\text { gaze(60Hz), Audio }(44.1 \mathrm{kHz})\end{array}$ \\
\hline Number of videos & 20 \\
\hline Self-report & $\begin{array}{l}\text { Emotional keywords, arousal, valence, dominance } \\
\text { and predictability }\end{array}$ \\
\hline Rating values & Discrete scale 1-9 \\
\hline
\end{tabular}

\section{PREPRoCESSING DATA AND FEATURES EXTRACTION}

Obtaining promising accuracies required different stages mainly the pre-processing data, features extraction and finally the classification step.

According to the experimental setup of the MAHNOB-HCI database, each trial contained 30 seconds before the beginning of the affective stimuli experience and another 30 seconds after the end. So firstly, we eliminated these two 30 seconds to have the pertinent information. Next, Butterworth filters were applied to eliminate artefacts and baseline wandering for the GSR, Resp and ECG signals. The cut-off frequencies are 0.3 $\mathrm{Hz}, 0.45 \mathrm{~Hz}$ and $0.5 \mathrm{~Hz}$, respectively.

Adding to characteristic features like the heart rate variability from the electrocardiogram (1) and the breathing rate from the respiratory volume, a bunch of statistical values were extracted from the data.

$$
\mathrm{HRV}=60 / \mathrm{t}_{\mathrm{R} \_\mathrm{R}}
$$
minute

Whereas: HRV is the heart rate variability in beats per

$$
\mathrm{t}_{\mathrm{R} \_\mathrm{R}} \text { : The mean of RR intervals }
$$

To reduce the difference between the participants, we normalized features by mapping each one to the interval $[0,1]$. The preprocessing data and features extraction stages were based on the studies reported in [21] and [20].

\section{SVM CLASSIFICATION}

Different machine learning algorithms were successfully applied to classify the human emotional states given a bunch of physiological features. We cite the artificial neural network (ANN), k-Nearest Neighbors (k-NN), Bayesian Network and Regression Tree (RT). In this approach, we employed the support vector machine which is the most popular and pertinent classifier in this issue [35]. Indeed, a comparative study described in [36], proved that the SVM gave the best accuracy rates rather than other machine learning techniques such as $\mathrm{k}$ $\mathrm{NN}$, regression tree and Bayesian network.
Basically, it is a supervised machine learning technique. Adding to linear classification, SVM resolves efficiently a nonlinear problem with its several kernels to obtain the optimized classification rates. SVM performs the classification by finding the suitable hyper-plans that separate the classes very well by maximizing the distance between each class and the hyperplans. For the implementation, we used the LibSVM library under MATLAB platform ${ }^{3}$ [37].

Tables.II and .III present the two and three defined classes using 1-9 discrete scales in arousal-valence areas. The rated scales were reported by the participant after/during watching the affective video. On the other hand, we also defined the three classes in arousal valence model using the nine affective keywords, which are (1) Joy or Happiness, (2) Amusement, (3) Sadness, (4) Disgust, (5) Anxiety, (6) Fear, (7) Surprise, (8) Anger, and (9) Neutral. According to the table .IV, we assigned the labels "High" and "Low" for arousal, "Positive" and "Negative" for valence. The three classes were "Clam", "Medium", and "Activated" for arousal and "Unpleasant", "Neutral" and "Pleasant" for valence dimension.

TABLE II. Two Classes IN ARousal-VALENCE Model

\begin{tabular}{|l|l|l|}
\hline Categorization & \multirow{2}{*}{ Rating Values “ $r$ " } \\
\hline Arousal & Valence & \\
\hline High & Negative & $\mathrm{r} \leq 4.5$ \\
\hline Low & Positive & $4.5 \leq \mathrm{r}$ \\
\hline
\end{tabular}

TABLE III. ThreE Defined Classes In Arousal-VALENCE Model

\begin{tabular}{|l|l|l|}
\hline \multicolumn{2}{|l|}{ Categorization } & \multirow{2}{*}{ Rating Values " $r$ " } \\
\hline Arousal & Valence & $1 \leq \mathrm{r} \leq 3$ \\
\hline Calm & Unpleasant & $4 \leq \mathrm{r} \leq 6$ \\
\hline Medium & Neutral & $7 \leq \mathrm{r} \leq 9$ \\
\hline Excited & Pleasant & \\
\hline
\end{tabular}

TABLE IV. DeFined Classes in ARousal-VALENCE Model using EMOTIONAL KEYWORDS

\begin{tabular}{|l|l|l|}
\cline { 2 - 3 } \multicolumn{1}{c|}{} & Affective Classes & Discrete Emotion Tagging \\
\hline \multirow{4}{*}{ Arousal } & Clam & Sadness, Disgust, Neutral \\
\cline { 2 - 3 } & Medium & Happiness(Joy), Amusement \\
\cline { 2 - 3 } & Activated & Surprise, Fear, Anger, Anxiety \\
\hline \multirow{4}{*}{ Valence } & Unpleasant & $\begin{array}{l}\text { Anger, Anxiety, Disgust, } \\
\text { Sadness, Fear }\end{array}$ \\
\cline { 2 - 3 } & Neutral & Neutral, Surprise \\
\cline { 2 - 3 } & Pleasant & Happiness(Joy), Amusement \\
\hline
\end{tabular}

\section{RESUlTS AND DISCUSSIONS}

In this section, we summarize and evaluate the obtained results for emotion classification in arousal valence dimension. We presented the emotional states in two and three defined classes, as explained earlier.

For the aim of this paper, we classified each peripheral physiological signal and then, we applied an early fusion for all descriptors to compare the proposed approach to related studies. The level feature fusion is to combine all the modalities before the training stage[38]. Thus, a simple concatenation was applied to all the extracted features. The table .V summarizes the classification accuracy after testing

\footnotetext{
${ }^{3}$ http://www.csie.ntu.edu.tw/_cjlin/libsvm/.
} 
several SMV's Kernel functions for two defined classes. In this table, we can clearly note that the ECG and the RESP signals are the most relevant signals for the emotion assessing task, and precisely ECG for arousal and RESP for valence.

We achieved $64.23 \%$ in arousal and $68.75 \%$ in valence dimension and these accuracies were very promising compared to related works. In fact, Koelstra et .al [21] obtained 62.7\% in valence 57\% in arousal and Torres Valencia et al.[39] achieved $55 \% \pm 3.9$ and $57.50 \% \pm 3.9$ in arousal and valence, respectively. Both of these previous studies used the DEAP database. The achieved results prove the potential of the recorded data in MAHNOB-HCI database and their chosen videos were more powerful to evoke the emotion than videos clips used in DEAP. This explanation is well developed in[34]. Indeed, the authors proved that the heart rate variability calculated from the ECG (not available in DEAP), is a very relevant feature in emotion recognition task and it is more accurate than the HRV calculated from the PPG signal which is recorded in DEAP database.

TABLE V. Classification Accuracies AFter using SEVERAl SVM's Kernel (Two Classes)

\begin{tabular}{|c|c|c|c|c|c|c|c|c|}
\hline & \multicolumn{8}{|c|}{ Classification Accuracies } \\
\hline & \multicolumn{2}{|l|}{ Linear } & \multicolumn{2}{|c|}{ Polynomial } & \multicolumn{2}{|l|}{ Sigmoid } & \multicolumn{2}{|c|}{ Gaussian } \\
\hline & Arousal & Valence & Arousal & Valence & Arousal & Valence & Arousal & Valence \\
\hline ECG & $65,03 \%$ & $60,13 \%$ & $65,73 \%$ & $60,83 \%$ & $62,10 \%$ & $65,03 \%$ & $66,4 \%$ & $58,74 \%$ \\
\hline GSR & $55,94 \%$ & $54,73 \%$ & $53,14 \%$ & $55,78 \%$ & $54,73 \%$ & $55,78 \%$ & $62,23 \%$ & $50,52 \%$ \\
\hline RESP & $61,05 \%$ & $62,10 \%$ & $60,83 \%$ & $61,05 \%$ & $60,13 \%$ & $53,84 \%$ & $65,03 \%$ & $62,10 \%$ \\
\hline Temp & $57,34 \%$ & $53,84 \%$ & $58,74 \%$ & $53,84 \%$ & $54,73 \%$ & $57,34 \%$ & $60,13 \%$ & $57,34 \%$ \\
\hline LFF & $63,63 \%$ & $65,03 \%$ & $64,23 \%$ & $60,83 \%$ & $60,83 \%$ & $57,34 \%$ & $63,63 \%$ & $68.75 \%$ \\
\hline
\end{tabular}

The table.VI presents the accuracies after classifying the emotion into three areas in arousal valence space using the selfreported scaling. On the other hand, Table.VII shows the results after defining the three classes basing on the nine selfreported affective keywords previously reported.

Both of these tables prove that the human's emotion is more noticeable from the respiration and electrocardiogram signals then other bodily responses (Temperature or Galvanic Skin Response). In addition, we can clearly see that the Gaussian kernel function is the best solution that could find the performed hyper-plans. Moreover, it is easier to recognize the emotion after fusing all the peripheral physiological signals, as shown in table V, VI and VII.

The table.VIII resumes the obtained results and three recent related works and it proves that the obtained accuracies are promising in the three ways for emotion's establishments in arousal-valence model.

The achieved accuracies are explained by the fact that we correctly pre-processed the signals to have the significant information. In addition, we warily selected features, which are relevant than chosen in the studies earlier mentioned [20][21][39].

TABLE VI. Classification Accuracies by using SEVERal SVM's Kernels (3 Classes using SCALing Rates)

\begin{tabular}{|c|c|c|c|c|c|c|c|c|}
\hline & \multicolumn{8}{|c|}{ Classification Rates } \\
\hline & \multicolumn{2}{|l|}{ Linear } & \multicolumn{2}{|c|}{ Polynomial } & \multicolumn{2}{|l|}{ Sigmoid } & \multicolumn{2}{|l|}{ Gaussian } \\
\hline & Arousal & Valence & Arousal & Valence & Arousal & Valence & Arousal & Valence \\
\hline ECG & $51,4 \%$ & $44,05 \%$ & $50 \%$ & $52,12 \%$ & $52,63 \%$ & $46,80 \%$ & $50,07 \%$ & $46,8 \%$ \\
\hline GSR & $47,36 \%$ & $48,93 \%$ & $48,42 \%$ & $48,93 \%$ & $49,37 \%$ & $45,74 \%$ & $50,52 \%$ & $47,87 \%$ \\
\hline Resp & $47,36 \%$ & $53,19 \%$ & $46,31 \%$ & $50 \%$ & $48,42 \%$ & $48,93 \%$ & $45,77 \%$ & $52 \%$ \\
\hline Temp & $40,14 \%$ & $43,3 \%$ & $42,95 \%$ & $45,45 \%$ & $45,26 \%$ & $51,05 \%$ & $42,25 \%$ & $45,45 \%$ \\
\hline LFF & $52,63 \%$ & $48,93 \%$ & $50,52 \%$ & $52,12 \%$ & $51,57 \%$ & $46,36 \%$ & $54,73 \%$ & $56,83 \%$ \\
\hline
\end{tabular}

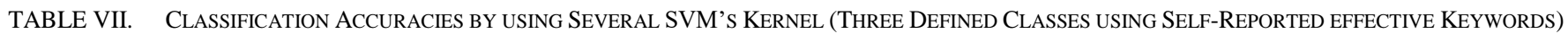

\begin{tabular}{|c|c|c|c|c|c|c|c|c|}
\hline & \multicolumn{8}{|c|}{ Classification Accuracies } \\
\hline & \multicolumn{2}{|l|}{ Linear } & \multicolumn{2}{|c|}{ Polynomial } & \multicolumn{2}{|l|}{ Sigmoid } & \multicolumn{2}{|c|}{ Gaussian } \\
\hline & Arousal & Valence & Arousal & Valence & Arousal & Valence & Arousal & Valence \\
\hline ECG & $44,21 \%$ & $48,42 \%$ & $43,15 \%$ & $49,47 \%$ & $50,52 \%$ & $48,42 \%$ & $45,26 \%$ & $51,57 \%$ \\
\hline GSR & $40 \%$ & $41,05 \%$ & $42,10 \%$ & $43,15 \%$ & $46,31 \%$ & $44,4 \%$ & $45,26 \%$ & $42,10 \%$ \\
\hline Resp & $51,57 \%$ & $49,47 \%$ & $52,63 \%$ & $46,31 \%$ & $47,36 \%$ & $48,42 \%$ & $49,47 \%$ & $44,21 \%$ \\
\hline Temp & $41,57 \%$ & $43,15 \%$ & $41,05 \%$ & $44,2 \%$ & $46,31 \%$ & $45,26 \%$ & $45,26 \%$ & $45,26 \%$ \\
\hline LFF & $48,42 \%$ & $48,42 \%$ & $50,52 \%$ & $54,73 \%$ & $46,31 \%$ & $49,47 \%$ & $\mathbf{5 9 , 5 7 \%}$ & $\mathbf{5 7 . 4 4 \%}$ \\
\hline
\end{tabular}


TABLE VIII. OBTAINED RESULTS COMPARED TO RELATED WORK FOR THE THREE WAYS IN MODELLING EMOTIONS

\begin{tabular}{|c|c|c|c|c|c|c|}
\hline & \multirow{2}{*}{\multicolumn{3}{|c|}{ Two Classes }} & \multicolumn{3}{|c|}{ Three Classes } \\
\hline & & & & \multirow{2}{*}{$\begin{array}{l}\text { Using 1-9 } \\
\text { scaling } \\
\text { values } \\
\text { Obtained } \\
\text { Results } \\
\end{array}$} & \multicolumn{2}{|c|}{$\begin{array}{l}\text { Using } 9 \text { emotional } \\
\text { keywords }\end{array}$} \\
\hline & $\begin{array}{l}\text { Obtained } \\
\text { Results }\end{array}$ & {$[21]$} & [39] & & $\begin{array}{l}\text { Obtained } \\
\text { Results }\end{array}$ & {$[20]$} \\
\hline Arousal & $64.23 \%$ & $57 \%$ & $\begin{array}{l}55.00 \% \\
\pm 3.9\end{array}$ & $54,73 \%$ & $59,57 \%$ & $46.2 \%$ \\
\hline Valence & $68.75 \%$ & $62.7 \%$ & $\begin{array}{l}57.50 \% \\
\pm 3.9 \\
\end{array}$ & $56,83 \%$ & $57.44 \%$ & $45.5 \%$ \\
\hline
\end{tabular}

\section{CONCLUSION}

This paper presented a whole process in emotion recognition system from peripheral physiological signals. For this aim, we used the recent multimodal database MAHNOBHCI. In this dataset, they collected the bodily responses from 24 participants after eliciting their feeling using 20 selected videos. Basing on the self-reported emotion from the participant, we proposed three ways to model the affective states in arousal valence space. In fact, we established two and three main classes using the discrete rating values (from 1 to 9 scales) and another model using 9 emotional keywords to define three areas in arousal valence dimension. We preprocessed the data to remove noise and artefacts from the data. Then, we extracted selected features. After normalizing them to minimize the difference between participants, an early level feature fusion was applied for further analysis. Finally, we classified for the first time each physiological signal and then the LFF data using the support vector machine. We used its different kernel's functions to perform the classification rates. Results showed the relevance of the electrocardiogram and respiration signals in emotion assessment task. Moreover, the RBF kernel is the most suitable algorithm. Results proved also, that detecting affective states is easier after fusing all the bodily responses. The obtained accuracies were promising compared to recent related works.

As future work, we aim to implement additional techniques such as the feature selection and reduction mechanisms (ANOVA, PCA, and Fisher) to eliminate the redundant information and select the most relevant features. Moreover, we would like to implement other classification algorithms that can lead for best results.

\section{ACKNOWLEDGMENT}

The authors of this paper would like to thank the MAHNOB-HCI's team for providing this freely multimodal database to develop this research ${ }^{4}$.

\section{REFERENCES}

[1] K. G. Smitha and A. P. Vinod, "Hardware efficient FPGA implementation of emotion recognizer for autistic children," IEEE International Conference on Electronics, Computing and Communication Technologies (CONECCT), pp. 1-4, Jan. 2013.
[2] Benoit et al., "Multimodal focus attention and stress detection and feedback in an augmented driver simulator," Pers. Ubiquitous Comput., vol. 13, no. 1, pp. 33-41, Jan. 2009.

[3] D. Katsis, N. Katertsidis, G. Ganiatsas, and D. I. Fotiadis, "Toward Emotion Recognition in Car-Racing Drivers: A Biosignal Processing Approach,” IEEE Trans. Syst. Man Cybern. - Part Syst. Hum., vol. 38, no. 3, pp. 502-512, May 2008.

[4] M. Paschero et al., "A real time classifier for emotion and stress recognition in a vehicle driver," IEEE International Symposium on Industrial Electronics , pp. 1690-1695, May 2012.

[5] Ghaderi, J. Frounchi, and A. Farnam, "Machine learning-based signal processing using physiological signals for stress detection," 22nd Iranian Conference on Biomedical Engineering (ICBME), pp. 93-98, November 2015.

[6] K. Rattanyu, M. Ohkura, and M. Mizukawa, "Emotion monitoring from physiological signals for service robots in the living space," in International Conference on Control Automation and Systems (ICCAS), pp. 580-583, Octobre 2010

[7] Suchitra, Suja P., and S. Tripathi, "Real-time emotion recognition from facial images using Raspberry Pi II," 3rd International Conference on Signal Processing and Integrated Networks (SPIN), pp. 666-670, February 2016.

[8] N. Chanthaphan, K. Uchimura, T. Satonaka, and T. Makioka, "Facial Emotion Recognition Based on Facial Motion Stream Generated by Kinect," 11th International Conference on Signal-Image Technology Internet-Based Systems (SITIS), pp. 117-124, November 2015.

[9] Turan, K.-M. Lam, and X. He, "Facial expression recognition with emotion-based feature fusion," Asia-Pacific Signal and Information Processing Association Annual Summit and Conference (APSIPA), pp. 1-6, December 2015.

[10] S. G. Koolagudi and K. S. Rao, "Emotion recognition from speech: a review," Int. J. Speech Technol., vol. 15, no. 2, pp. 99-117, Jun. 2012.

[11] F. Chenchah and Z. Lachiri, "Acoustic Emotion Recognition Using Linear and Nonlinear Cepstral Coefficients," Int. J. Adv. Comput. Sci. Appl., vol. 6, no. 11, 2015.

[12] S. Wioleta, "Using physiological signals for emotion recognition," 6th International Conference on Human System Interactions (HSI), pp. 556561, June 2013.

[13] R. W. Picard, E. Vyzas, and J. Healey, "Toward machine emotional intelligence: analysis of affective physiological state," IEEE Trans. Pattern Anal. Mach. Intell., vol. 23, no. 10, pp. 1175-1191, October 2001

[14] Y. Velchev, S. Radeva, S. Sokolov, and D. Radev, "Automated estimation of human emotion from EEG using statistical features and SVM," Digital Media Industry Academic Forum (DMIAF), pp. 40-42, July 2016.

[15] Y. Gu, K.-J. Wong, and S.-L. Tan, "Analysis of physiological responses from multiple subjects for emotion recognition," IEEE 14th International Conference on e-Health Networking, Applications and Services (Healthcom), pp. 178-183, October 2012.

[16] S. Basu et al., "Emotion recognition based on physiological signals using valence-arousal model," Third International Conference on Image Information Processing (ICIIP), pp. 50-55, December 2015.

[17] S. Thushara and S. Veni, "A multimodal emotion recognition system from video," International Conference on Circuit, Power and Computing Technologies (ICCPCT), pp. 1-5, March 2016.

[18] A. Torres, Á. A. Orozco, and M. A. Álvarez, "Feature selection for multimodal emotion recognition in the arousal-valence space," 35th Annual International Conference of the IEEE Engineering in Medicine and Biology Society (EMBC), pp. 4330-4333, July 2013.

[19] M. Soleymani, J. Lichtenauer, T. Pun, and M. Pantic, "A Multimodal Database for Affect Recognition and Implicit Tagging," IEEE Trans. Affect. Comput., vol. 3, no. 1, pp. 42-55, Jan. 2012.

[20] S. Koelstra et al., "DEAP: A Database for Emotion Analysis;Using Physiological Signals," IEEE Trans. Affect. Comput., vol. 3, no. 1, pp. 18-31, Jan. 2012.

\footnotetext{
${ }^{4}$ https://mahnob-db.eu/hci-tagging
} 
[21] G. N. Yannakakis, K. Isbister, A. Paiva, and K. Karpouzis, "Guest Editorial: Emotion in Games," IEEE Trans. Affect. Comput., vol. 5, no. 1, pp. 1-2, Jan. 2014.

[22] Jonghwa Kim and E. Andre, "Emotion recognition based on physiological changes in music listening," IEEE Trans. Pattern Anal. Mach. Intell., vol. 30, no. 12, pp. 2067-2083, Dec. 2008.

[23] Jonghwa Kim and E. Andre, "Emotion recognition based on physiological changes in music listening," IEEE Trans. Pattern Anal. Mach. Intell., vol. 30, no. 12, pp. 2067-2083, Dec. 2008.

[24] J. Fleureau, P. Guillotel, and Q. Huynh-Thu, "Physiological-Based Affect Event Detector for Entertainment Video Applications," IEEE Trans. Affect. Comput., vol. 3, no. 3, pp. 379-385, Jul. 2012.

[25] "Sentiment Symposium Tutorial: Language and cognition." [Online]. Available: http://sentiment.christopherpotts.net/lingcog.html. [Accessed: 19-Feb-2017].

[26] S. M, J. A. Russell, and L. F. Barrett, "Structure of self-reported current affect: Integration and beyond," J. Pers. Soc. Psychol., vol. 77, no. 3, pp. 600-619, 1999.

[27] H. Xu and K. N. Plataniotis, "Subject independent affective states classification using EEG signals," IEEE Global Conference on Signal and Information Processing (GlobalSIP), pp. 1312-1316, December 2015 .

[28] "Emotion, core affect, and psychological construction." [Online]. Available:

https://www.researchgate.net/publication/276947577_Emotion_core_aff ect_and_psychological_construction. [Accessed: 17-Nov-2016].

[29] P. Ekman and D. Cordaro, "What is Meant by Calling Emotions Basic," Emot. Rev., vol. 3, no. 4, pp. 364-370, October 2011.

[30] G. McKeown, M. F. Valstar, R. Cowie, and M. Pantic, "The SEMAINE corpus of emotionally coloured character interactions," IEEE
International Conference on Multimedia and Expo, pp. 1079-1084, July 2010 .

[31] M. Grimm, K. Kroschel, and S. Narayanan, "The Vera am Mittag German audio-visual emotional speech database," IEEE International Conference on Multimedia and Expo , pp. 865-868, June 2008.

[32] J. A. Healey and R. W. Picard, "Detecting Stress During Real-World Driving Tasks Using Physiological Sensors," IEEE Trans. Intell. Transp. Syst., vol. 6, no. 2, pp. 156-166, Jun 2005.

[33] C. Godin, F. Prost-Boucle, A. Campagne, S. Charbonnier, S. Bonnet, and A. Vidal, "Selection of the most relevant physiological features for classifying emotion," Proceedings in International conference on physiological computing systems (PhyCS), January 2015.

[34] C. J. C. Burges, "A Tutorial on Support Vector Machines for Pattern Recognition,” Data Min. Knowl. Discov., vol. 2, no. 2, pp. 121-167.

[35] Changchun Liu, P. Rani, and N. Sarkar, "An empirical study of machine learning techniques for affect recognition in human-robot interaction," IEEE/RSJ International Conference on Intelligent Robots and Systems, pp. 2662-2667, August 2005.

[36] C.-C. Chang and C.-J. Lin, "LIBSVM: A library for support vector machines," ACM Trans. Intell. Syst. Technol., vol. 2, no. 3, pp. 1-27, Apr. 2011.

[37] Z. Guendil, Z. Lachiri, C. Maaoui, and A. Pruski, "Emotion recognition from physiological signals using fusion of wavelet based features," in 2015 7th International Conference on Modelling, Identification and Control (ICMIC), pp. 1-6, December 2015.

[38] C. A. Torres-Valencia, H. F. Garcia-Arias, M. A. A. Lopez, and A. A. Orozco-Gutierrez, "Comparative analysis of physiological signals and electroencephalogram (EEG) for multimodal emotion recognition using generative models," Signal Processing and Artificial Vision 2014 XIX Symposium on Image, pp. 1-5, September 2014. 